\title{
Topology Tracking for the Visualization of Time-Dependent Two-Dimensional Flows
}

\author{
X. Tricoche, T. Wischgoll, G. Scheuermann, H. Hagen \\ University of Kaiserslautern, P.O. Box 3049, D-67653 Kaiserslautern, Germany \\ E-mail: $\{$ tricoche|wischgol|scheuer|hagen\} @informatik.uni-kl.de
}

\begin{abstract}
The paper presents a topology-based visualization method for time-dependent two-dimensional vector fields. A time interpolation enables the accurate tracking of critical points and closed orbits as well as the detection and identification of structural changes. This completely characterizes the topology of the unsteady flow. Bifurcation theory provides the theoretical framework. The results are conveyed by surfaces that separate subvolumes of uniform flow behavior in a three-dimensional space-time domain.
\end{abstract}

\section{Introduction}

Topology-based visualization of steady two-dimensional vector fields was initiated by Helman and Hesselink [3]. Its basic principle however was already familiar to the fluid dynamics community [1]. Critical points - where the field is zero - and closed orbits are located and classified. Further, special streamlines are extracted that sketch the structure of the flow. This method has been widely applied in the last decade even if the visualization of closed orbits is a recent advance [12]. Now in the case of unsteady vector fields, the original method must still be extended to offer insight into the structural evolution of the flow over time. A simple graphical connection of the topological graphs obtained for successive discrete time steps [4] is not satisfying. This approach lacks time continuity which prevents the resulting description from properly identifying and depicting qualitative changes that occur between discrete time steps. Indeed, time as additional parameter entails the occurrence of new topological features called bifurcations. They correspond to continuous transitions between stable structures. Now, the visualization of these features is of major interest because they show how a vector field evolves to the structures obtained on discrete time samples by conventional methods. Hence their depiction requires the analysis of the vector field as a continuous map defined over a spacetime domain. This is the basic idea of the presented method.

The paper is structured as follows. First we briefly review previous work dealing 
with the visualization of time-dependent flows. Then we recall fundamental definitions of vector field topology and provide an overview of typical bifurcations (section 3). Next we describe in section 4 how time interpolation is processed to obtain the required continuous space-time map. This enables the precise tracking of critical points over time as explained in section 5. Finally the surfaces spanned by the curves of the topological graph are constructed (see section 6). Results are shown in section 7 .

\section{Related Work}

As far as unsteady flows are concerned, a very comprehensive introduction to bifurcation theory is proposed in [2]. This work is part of the Visual Math Project that explains the basic mathematical notions by means of discerning sketches. Two of the authors [12] presented an algorithm that computes streamlines in the 2D steady case while detecting if they run into a closed streamline. This can also be used in time slices of a time-dependent dataset. Hepting et al. [8] study invariant tori in four-dimensional dynamical systems by using suitable projections into three dimensions to enable detailed visual analysis of the tori. Wegenkittel et al. [10] present visualization techniques for known features of dynamical systems. Furthermore, the practical significance of unsteady flows has led to several techniques for the visualization of time-dependent vector fields. In his paper [5], Dickinson describes a method for interactive analysis of the topology of time-dependent vector fields. $2 \mathrm{D}$ and $3 \mathrm{D}$ are both considered. The main point here is the correlation of the separatrices between two consecutive time steps. A method for computing streaklines in 3D unsteady flow fields has been proposed in [6]. The basic principle is to integrate streaklines thanks to an interpolation over the $3 \mathrm{D}$ space and time. The technique works also with moving grids. Using this scheme, a method for displaying unsteady flow volumes has been presented in [7]. Based upon an adaptive subdivision strategy, the authors arrive at integrating streaklines starting on a generating polygon. For feature visualization, a scheme is proposed in [9] to track and correlate the extracted structures by detecting the following fundamental events: Continuation, bifurcation, amalgamation, creation and dissipation.

\section{Time-Dependent Topology}

The topology of a steady vector field is determined by critical points, associated separatrices and closed orbits. Turning to the unsteady case local and global bifurcations must be considered. These notions are reviewed next. 


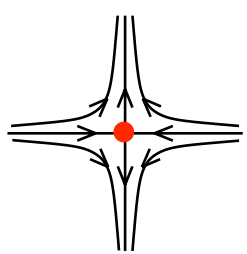

Saddle Point:

$\mathrm{R} 1<0, \mathrm{R} 2>0$,

$\mathrm{I} 1=\mathrm{I} 2=0$

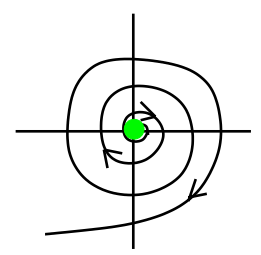

Repelling Focus: $\mathrm{R} 1=\mathrm{R} 2>0$, $\mathrm{I} 1=-\mathrm{I} 2<>0$

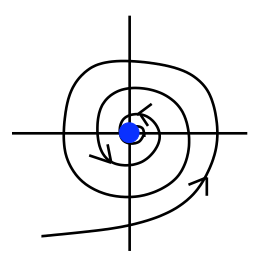

Attracting Focus:

\begin{abstract}
$\mathrm{R} 1=\mathrm{R} 2<0$
\end{abstract}
$\mathrm{I} 1=-\mathrm{I} 2<>0$

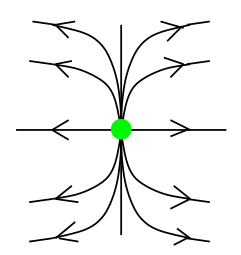

Repelling Node:

$\mathrm{R} 1, \mathrm{R} 2>0$, $\mathrm{I} 1=\mathrm{I} 2=0$

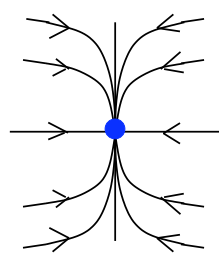

Attracting Node:

$\mathrm{R} 1, \mathrm{R} 2<0$,

$\mathrm{I} 1=\mathrm{I} 2=0$

Fig. 1. Common first order singularity types

\subsection{Critical Points and Separatrices}

Critical points (also called singularities) are the only locations where streamlines can intersect. They exist in various types that correspond to specific geometries of the streamlines in their neighborhood. We focus on the linear case: There are 5 common types characterized by the eigenvalues of the Jacobian (see Fig. 1). Attracting nodes and foci are sinks while repelling nodes and foci are sources. A fundamental invariant is the so-called index of a critical point, defined as the number of field rotations while traveling around the critical point along a closed curve, counterclockwise. Note that all sources and sinks mentioned above have index +1 while saddle points have index -1 . Separatrices are streamlines that start or end at a saddle point.

\subsection{Closed Streamlines}

A closed streamline, which is sometimes known as closed orbit, is a streamline that is connected to itself so that a loop is built. Consequently, this is a streamline $c_{a}$, so that there is a $t_{0} \in \mathbb{R}$ with $c_{a}\left(t+n t_{0}\right)=c_{a}(t) \quad \forall n \in \mathbb{N}$. From a topological point of view, closed streamlines behave in the same way as sources or sinks. To detect these closed streamlines we use the algorithm proposed by two of the authors [12]. Interpolating linearly on the given grid we get a continuous vector field. To find closed streamlines we use the underlying grid to find a region that is never left by the streamline. If there is no critical point inside this region, we have found a closed streamline according to the Poincaré-Bendixson-theorem.

\subsection{Bifurcations}

One distinguishes two types of structural transitions: local and global bifurcations. In the following, we focus on typical 2D local bifurcations and present a typical aspect of global bifurcations. 


\subsubsection{Local Bifurcations}

There are two main types of local bifurcations affecting the nature of a singular point in 2D vector fields. The first one is the so-called Hopf bifurcation. The second one is the pairwise annihilation or creation of a saddle and a source or sink, called fold bifurcation. Other local bifurcations (like those where an attracting and a repelling closed orbit appear simultaneously for instance) may also occur. Yet, for our purpose, we only pay attention to those that concern singularities.
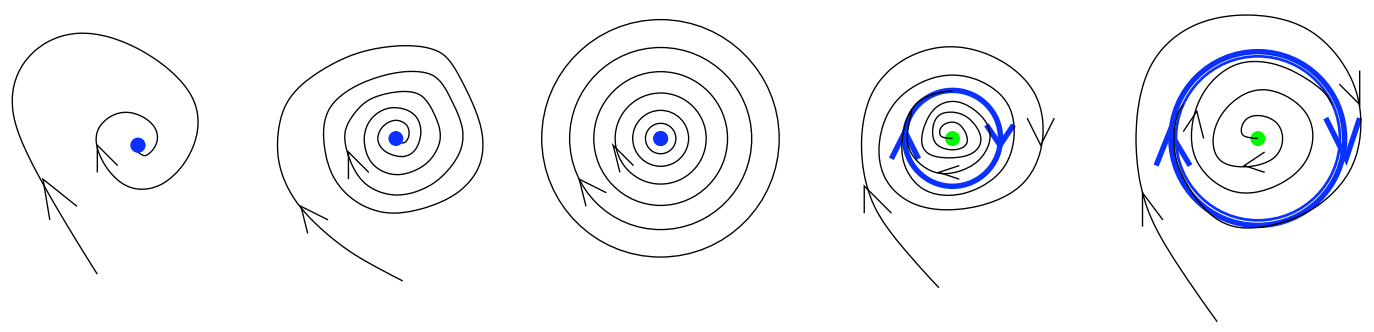

Fig. 2. Hopf bifurcation

3.3.1.1 Hopf Bifurcation One starts with an attracting focus. If the attracting effect of this sink weakens, the number of streamline rotations around this critical point increases (the convergence "slows down"). At the bifurcation point, one obtains a center point, which is an unstable structure. Then a new stable structure appears which consists of an attracting closed orbit moving away from the critical point. The critical point itself has transformed into a repelling focus (i.e. a source). So, a sink has changed into a source with the emission of a cycle. An illustration of this evolution is given in Fig. 2. The reverse process is possible too. Similar transitions are obtained by replacing sources by sinks and vice versa.

3.3.1.2 Fold Bifurcations At the beginning, there are a saddle point and a singularity of index +1 , say a sink, linked by a separatrix. If the attraction/repulsion relation between both singularities along the separatrix weakens, both critical points become closer and closer until they merge. At this point, an unstable critical point appears. As time goes on, this unstable structure vanishes: The new stable structure contains no singularity: This is a pairwise annihilation or fold catastrophe (see Fig. 3). Again the reverse evolution can occur as well. Similar transitions are obtained by inverting the direction of the flow (the sink becomes a source).

\subsubsection{Global Bifurcations}

As opposed to the cases mentioned above, global bifurcations do not take place in a small neighborhood of a singularity but entail significant changes in the flow structure and involve large domains. We shall not go into details but mention a typical configuration exhibited by such transitions: The unstable saddle-saddle connection. 

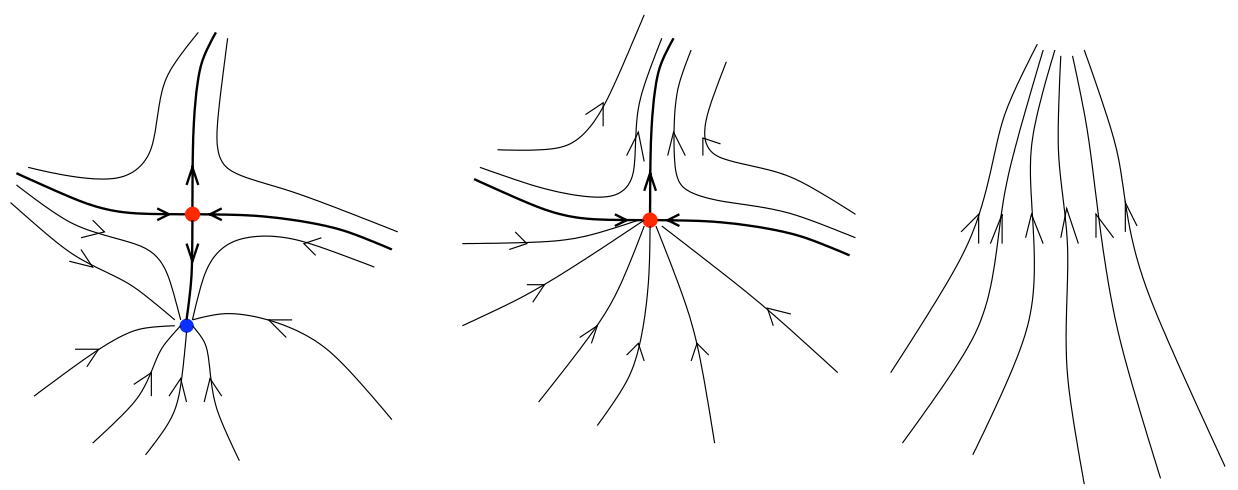

Fig. 3. Pairwise annihilation

One distinguishes heteroclinic separatrices that link two saddle points and homoclinic separatrices that connect a saddle point to itself.

The former is the central constituent of basin bifurcations. Consider Fig. 4. One
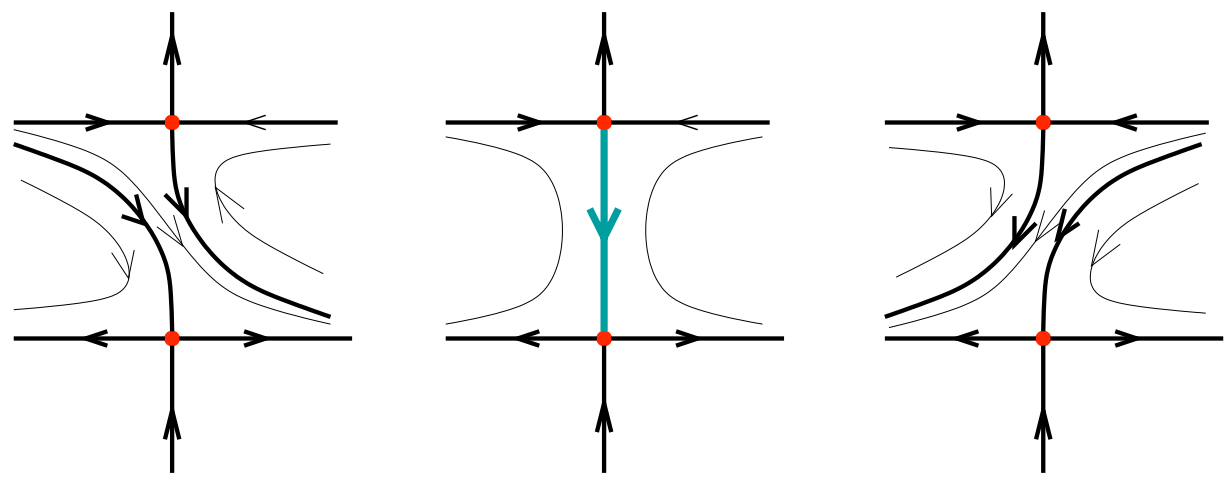

Fig. 4. Basin bifurcation

starts with two saddle points that are not connected. This is a stable structure. Progressively, two separatrices become closer and closer until they meet: Both saddle points are connected through a heteroclinic separatrix. Right after this moment, the separatrix splits into two distinct separatrices which constitutes a swap, compared to the initial configuration.

Homoclinic separatrices occur in so-called Periodic Blue Sky Bifurcations. Here there are two different types of critical points involved: a saddle and an attracting focus. Figure 5 shows the situation. As the attracting effect of the focus gets weaker we see a homoclinic connection after some time where the saddle is connected to itself. This results in a bifurcation: when this configuration breaks up again we find a limit cycle which simply appears out of the blue. The reason for the occurrence of the closed streamline is that the attracting focus is totally unaffected by the whole event. Since there is an outflow to the critical point inside and to the saddle there must be a critical point or a closed streamline in this region according to the Poincaré-Bendixson theorem. Because there are only two critical points a closed streamline emerged. 


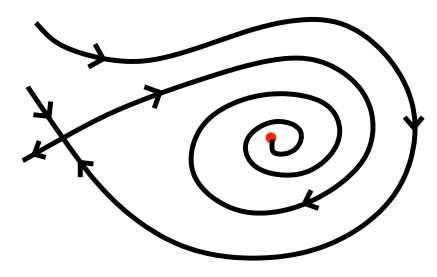

(a)

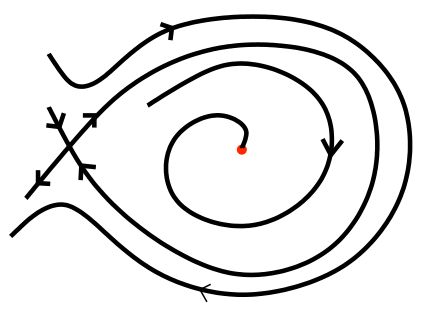

(b)

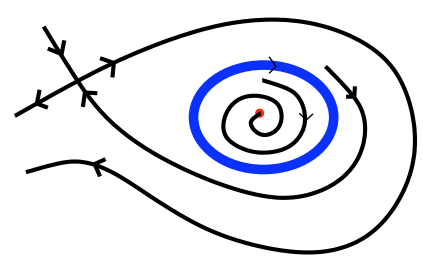

(c)

Fig. 5. Periodic Blue Sky in 2D bifurcation

\section{Data Representation}

The visualization of time dependent vector data has to deal with a higher dimensional mathematical space where time is an additional dimension. This space must be handled as a continuous one to permit the detection and depiction of fundamental features like bifurcations. In our 2D case, time is considered as third coordinate axis and the whole data embedded in a 3D grid.

\subsection{Grid Construction}

Practically, we process a $2 \mathrm{D}$ vector field lying on a triangulation with constant vertices over time. We place the several instantaneous states of the field parallel to another (each of them is called time plane in the following) with respect to their position along the time line. A 3D grid evolves by connecting these planar grids together with prism cells that link corresponding triangles as shown in Fig. 6. The choice of this cell type is explained in the following.

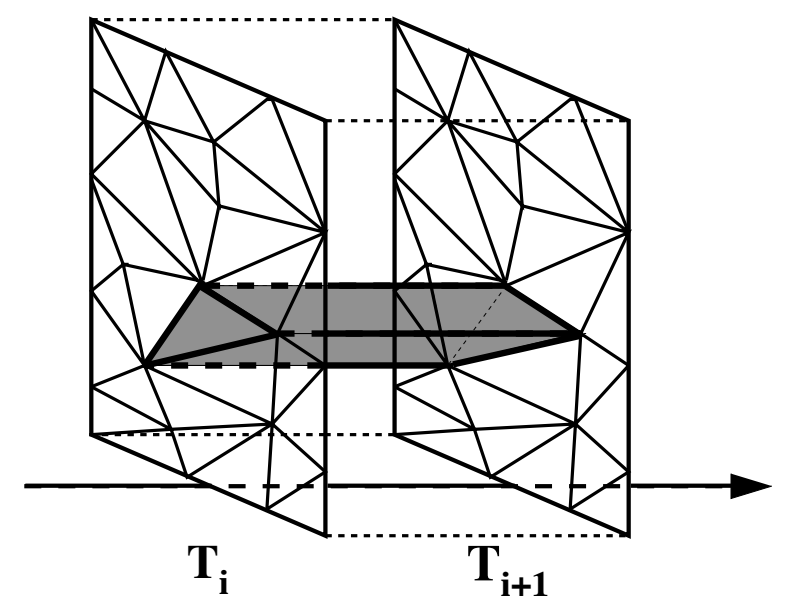

Fig. 6. Prism cell 


\subsection{Interpolation Scheme}

The input data are 2D vectors. Our task consists in interpolating a 2D vector field over a 3D grid. We suppose a piecewise linear interpolation over each planar triangulation associated with a time step. We introduce a piecewise linear time interpolation. It is defined in each prism as follows. For a given prism cell lying between $t=t_{i}$ and $t=t_{i+1}$, let

$$
\overrightarrow{f_{j}}(x, y)=\overrightarrow{a_{j}}+\overrightarrow{b_{j}} x+\overrightarrow{c_{j}} y, \quad j \in\{i, i+1\}
$$

be the affine linear interpolants corresponding to the triangle faces lying in the planes $\left\{t=t_{i}\right\}$ and $\left\{t=t_{i+1}\right\}$ respectively. The vector value at a position $(x, y, t)$ is given by

$$
\vec{f}(x, y, t)=\vec{a}(t)+\vec{b}(t) x+\vec{c}(t) y
$$

where $\vec{a}(t)$ is linearly interpolated between $\overrightarrow{a_{i}}$ and $\overrightarrow{a_{i+1}}$, idem for $\vec{b}(t)$ and $\vec{c}(t)$. Remark that this formula ensures, for each fixed value $t \in\left[t_{i}, t_{i+t}\right]$, that $\vec{f}_{t}$ is affine linear in $x$ and $y$.

\section{Feature Tracking}

Now we track critical points and closed orbits over time, using the interpolation scheme presented above. In particular, we seek the local bifurcations that may modify the topological structure: fold and Hopf bifurcations. To reduce the memory needs of our method, we do not process the whole grid at once but only consider two consecutive time planes and handle the prism cells connecting them, forming a "time slice". We first determine for each cell the possible entry and exit positions of singular points and then reconnect these pieces of information to track every singularity from one time plane to the next. Then we track possible closed orbits along the sink or source paths from the Hopf bifurcations we found.

\subsection{Cell-Wise Singularity Tracking}

The interpolant has been chosen to contain at most one critical point for a fixed value of $t$ in a cell. This is due to the affine linear nature of its restriction to any time plane. Straightforward calculus leads to the following singularity coordinates ( $\times$ denotes the cross product of $2 \mathrm{D}$ vectors).

$$
\left\{\begin{array}{l}
x(t)=(\vec{c}(t) \times \vec{a}(t)) /(\vec{b}(t) \times \vec{c}(t)) \\
y(t)=(\vec{a}(t) \times \vec{b}(t)) /(\vec{b}(t) \times \vec{c}(t))
\end{array}\right.
$$


If $t$ moves from $t_{i}$ to $t_{i+1}$, the singularity position describes a $3 \mathrm{D}$ curve. Yet, we are only interested in the curve sections that intersect the interior domain of the considered prism cell. A simple way to determine them is to compute the position of the singularities lying on the faces of the prism. We sort the (3D) positions found in ascending time order. We associate them pairwise and identify for each pair an entry and an exit position.

We next identify the singularity types to complete the topological information. The generic types of stable singularities are source, sink or saddle. The transition from one type to another is a bifurcation. From section 3 we consider two bifurcation types: fold and Hopf bifurcation. Since a prism cell contains at most one critical point for a given $t$, no fold bifurcation can occur inside it: A fold bifurcation must occur on the cell boundary. Consequently, we only seek Hopf bifurcations occurring between two consecutive entry and exit points. More precisely, since the determination of a singularity type is based upon the eigenvalues of the Jacobian we compute them at each entry and exit point and check if they are the same. If not, a Hopf bifurcation is on the way. Because a Hopf bifurcation corresponds to a transition from a repelling to an attracting nature, the instantaneous nature of the intermediate singularity is a center and the trace of the Jacobian is zero at this point, which we use as criterion to determine the exact time location of the bifurcation. It follows

$$
t=t_{i}+\left(t_{i+1}-t_{i}\right) \cdot \frac{-\left(b_{i}^{0}+c_{i}^{1}\right)}{b_{i+1}^{0}-b_{i}^{0}+c_{i+1}^{1}-c_{i}^{1}} .
$$

The corresponding position $(x(t), y(t), t)$ is the location of a Hopf bifurcation.

\subsection{Global Singularity Tracking}

At this stage, every cell knows the successive entry and exit positions of the critical point paths through its interior domain, as well as the possible presence of a Hopf bifurcation on the way. Yet, this information is scattered and must be reconnected to offer a global view of the topology evolution over time. A fundamental aspect of this task is to find and identify the bifurcations that may take place on the faces. They are detected during the reconnection of singularity paths lying in neighbor cells as we show next.

The reconnection is done in a single time slice at once. Basically we want to track any critical point located in the first time plane until it either reaches the next, vanishes or transforms through a bifurcation. To explain the method we consider the example shown in Fig. 7 . We start with a sink in $t_{i}$. The information collected in the corresponding cell provides us with an exit position. This position is an entry position for the neighboring prism. Now, the type of the singularity in this second cell is not necessarily the same as in the first one by discontinuity of the Jacobian in piecewise linear fields. Here, the sink has become a source. Consequently, the 


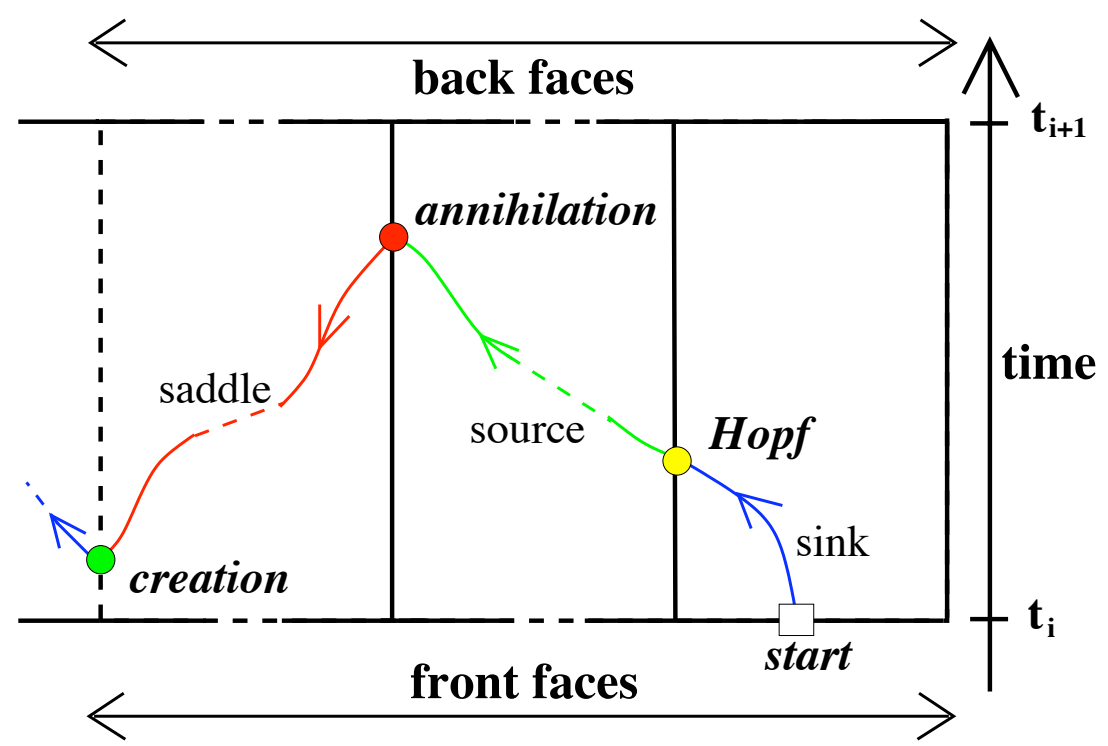

Fig. 7. Possible cases when leaving a prism cell through a rectangular face

current position is the location of a Hopf bifurcation. If we proceed in the same way we will eventually reach a position on a face corresponding to a source in the one cell and to a saddle in the other. Furthermore this position is an exit position for both cells. This characterizes a pairwise annihilation since the saddle and the source have merged and no critical point is present afterward. If we proceed with the saddle point we have to track the singularity backwards (see arrows along the paths). In our example, this leads to a position on a face that corresponds to a saddle in the one cell and to a sink in the other. Here, the position is an entry on both sides: We have found a pairwise creation. Further tracking in the same manner leads eventually to an exit position lying either on the next time plane $t_{i+1}$ or on the current one $t_{i}$. If this is done for every critical point starting in $t_{i}$, we track singularities backward from $t_{i+1}$.

In fact this simple example presents all possible cases in the context of our method (obviously the fold and Hopf bifurcations encountered here can occur in any form, e.g. pairwise annihilation of saddle and sink or Hopf bifurcation from source to sink and Hopf bifurcations may be found in the interior of a prism, according to what precedes). As a matter of fact, the piecewise linearity of the vector field at any fixed time $t$ prohibits other configurations. In particular, critical points of same type cannot be present in neighboring cells (this is a simple index property [11]) and therefore do not merge. Moreover, local consistency forbids the transformation of a saddle in a sink (or source) and vice versa since the index of the corresponding region must remain constant. Further details can be found in [13]. 


\subsection{Closed Streamlines}

After we tracked the singularity paths, we analyze the vector field in discrete timesteps. Since there must be a critical point inside each closed streamline we use the critical point path containing a Hopf bifurcation as a starting point for our streamline algorithm which detects the closed streamline if it exists. Therefore we follow the critical point path in discrete steps in positive and negative directions starting at the bifurcation. After we detect a closed streamline we have to check if it really surrounds the critical point. This is necessary because the streamline may have run in another closed streamline in a totally different region of the flow. Obviously, closed streamlines surrounding the critical point occur only in one of the two temporal directions. This process continues until the closed streamlines reach either another bifurcation which breaks them up or the boundary of the grid.
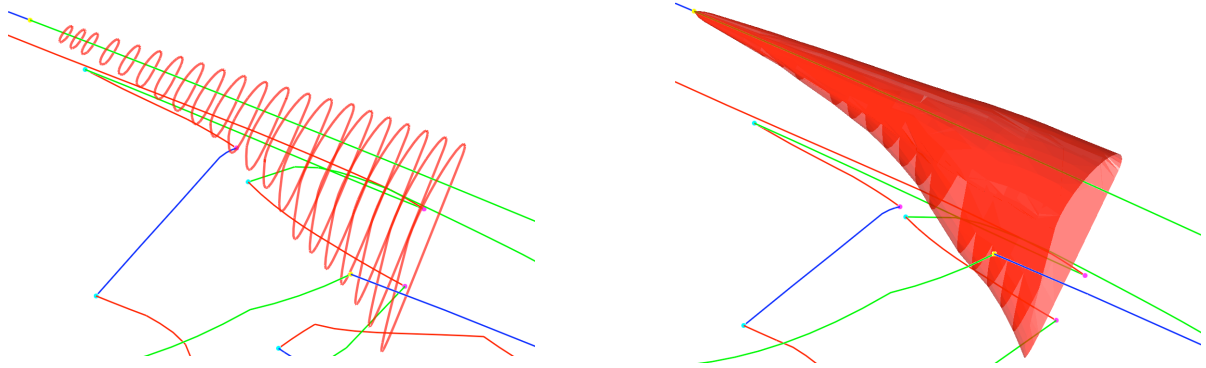

Fig. 8. Closed streamlines evolving over time.

Figure 8 shows on the left the result of this step, where we have found the closed streamlines at various time steps. The closed streamlines are approximated by several line segments. The Hopf bifurcation, where we started to detect the closed streamlines, is marked with a yellow point. In this example the life cycle of the closed streamline is started by a Hopf bifurcation and terminated by a Periodic Blue Sky in 2D bifurcation. To visualize the evolution of closed streamlines, we construct tubes from the various closed streamlines similar to the pictures by Abraham and Shaw [2]. The result is shown in figure 8 on the right. The concatenation of the singularity paths and the tubes built by the closed streamlines result in an abstraction of the path of the singularity.

\section{Structure Tracking}

In the $2 \mathrm{D}$ steady case, separatrices are curves that emanate from saddle points. As the saddle points move through the $2 \mathrm{D}$ /time grid, so do their corresponding separatrices, describing separating surfaces. These surfaces are essential to understand the structural evolution of unsteady vector fields. As a matter of fact, they determine the instantaneous global structure of the associated flow since they provide the edges of the topological graph. Therefore the analysis of their behavior is re- 
quired to identify structural changes that affect the qualitative nature of complete regions. From what precedes we know that such changes may correspond to global bifurcations. They may also be caused by cycle bifurcations that were not detected so far since we concentrated on the paths of critical points.

To compute and depict these surfaces, we can make use of the whole information gained about the singular points in the tracking step. Practically, given a saddle path, we save at its first position in time and for each of its four separatrices the following information: Starting vector, associated direction (converging/diverging) and reference of the singular path reached or last position obtained by numerical integration and corresponding case: boundary (grid boundary reached) or cycle (a closed streamline was detected by our algorithm). At the next position along the saddle path, we associate every separatrix with one at the previous position by taking its best approximation in terms of starting vector and direction. After that, we check if both corresponding separatrices reach the same singular path, the same cycle or close positions on the grid boundary. In this case, we add the new separatrix to the surface spanned by the previous ones (we add a new "ribbon" to this surface). Otherwise, we check if the path reached previously has ended at a bifurcation point. In this case, we terminate the previous surface at the bifurcation by integrating a separatrix from its exact time position and start a new one at the current separatrix. If no local bifurcation has occurred but connectivity has changed, we face either a global or a cycle bifurcation that could not be detected during singularity tracking: We simply end the surface at the previous separatrix and start a new surface at the current separatrix. Doing this for each discrete position along the saddle path, and that for each saddle path, we are able eventually to depict all separating surfaces in the domain.

\section{Results}

To test our method, we have created an analytic vector field containing four critical points, whose positions are functions of time, describing closed curves in the plane. We have sampled this vector field on a rectilinear point set for several values of the time parameter. The rotation of the critical points entails many structural changes for the topology, which is very interesting for our purpose since bifurcations are present and may be observed with our method. We show first the results of the singularity tracking step: The path of each critical point through time has been tracked as well as all the local bifurcations taking place (indicated by small balls): See Fig. 9. If one focuses on a particular bifurcation, one can observe how the separatrices evolve through the bifurcation point. In the case of a Hopf bifurcation for instance, we consider the picture without separatrix surfaces drawn (see Fig. 10). The creation of a closed orbit marked as a red tube can be easily seen. Fig. 11 shows a Periodic Blue Sky in 2D bifurcation which is terminated by a saddle singularity. 


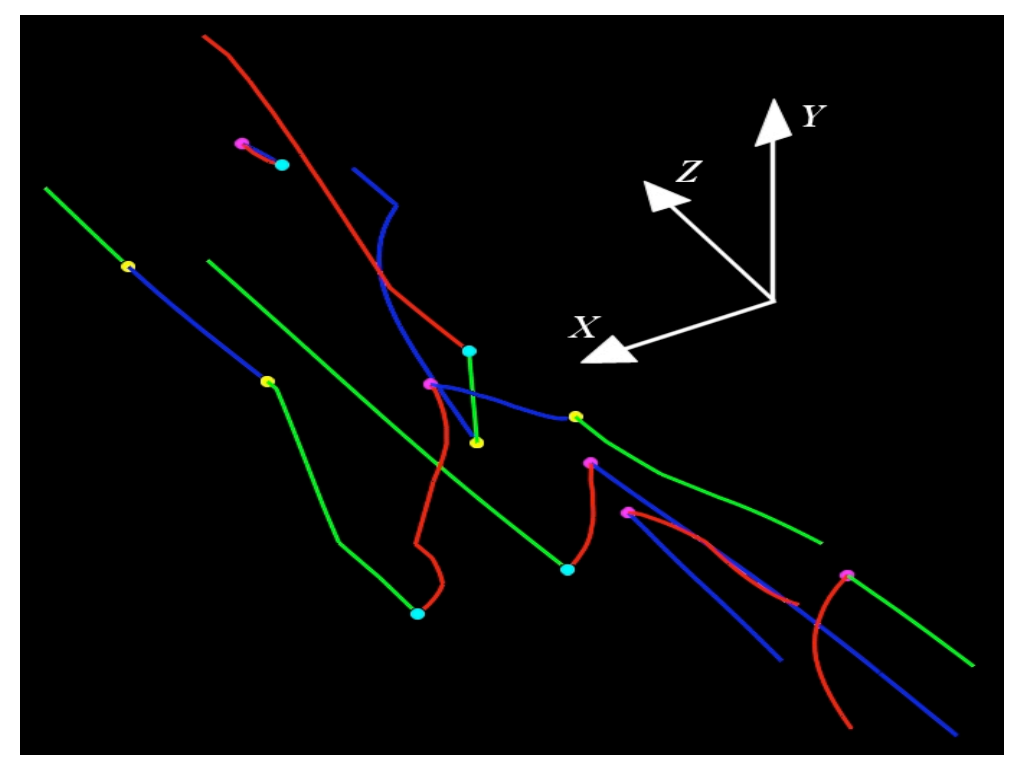

Fig. 9. Singularities' path through time and associated bifurcations

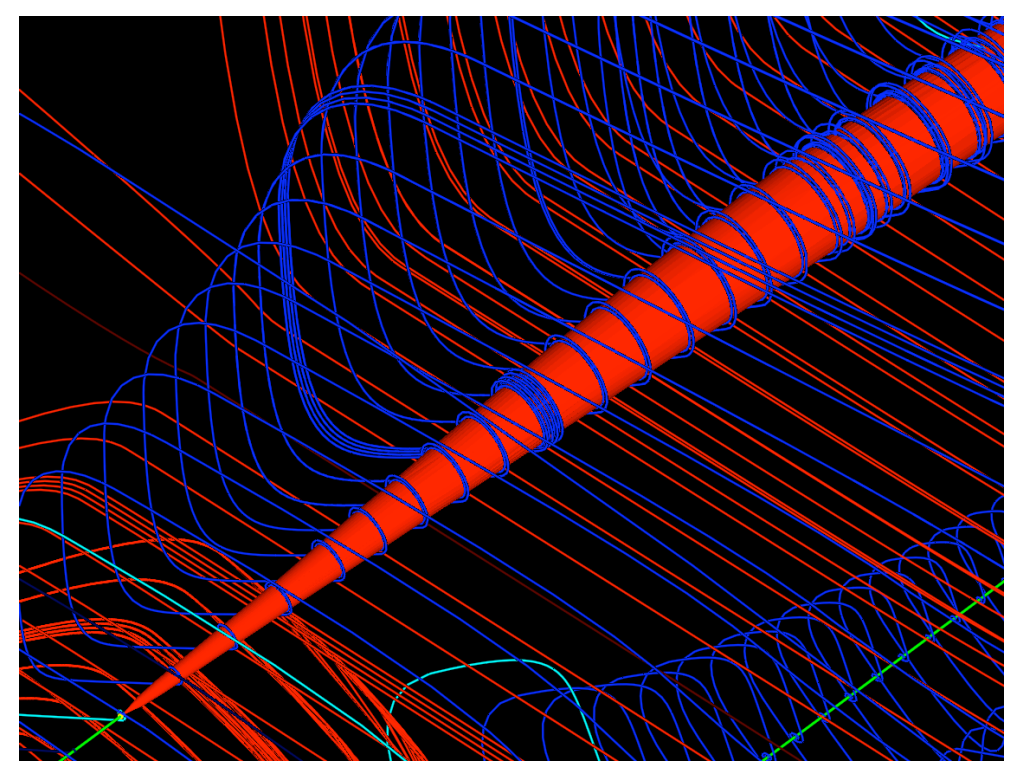

Fig. 10. Hopf bifurcation

At last, as an illustration of the topological pictures our method can produce, Fig. 12 proposes an overview of the whole topology evolution in the same perspective as in Fig. 9. The breaks that can be observed on the surfaces correspond to structural transitions associated with bifurcations. The two colors used for the surface depiction refer to the stable and unstable directions of the saddle points, respectively. 


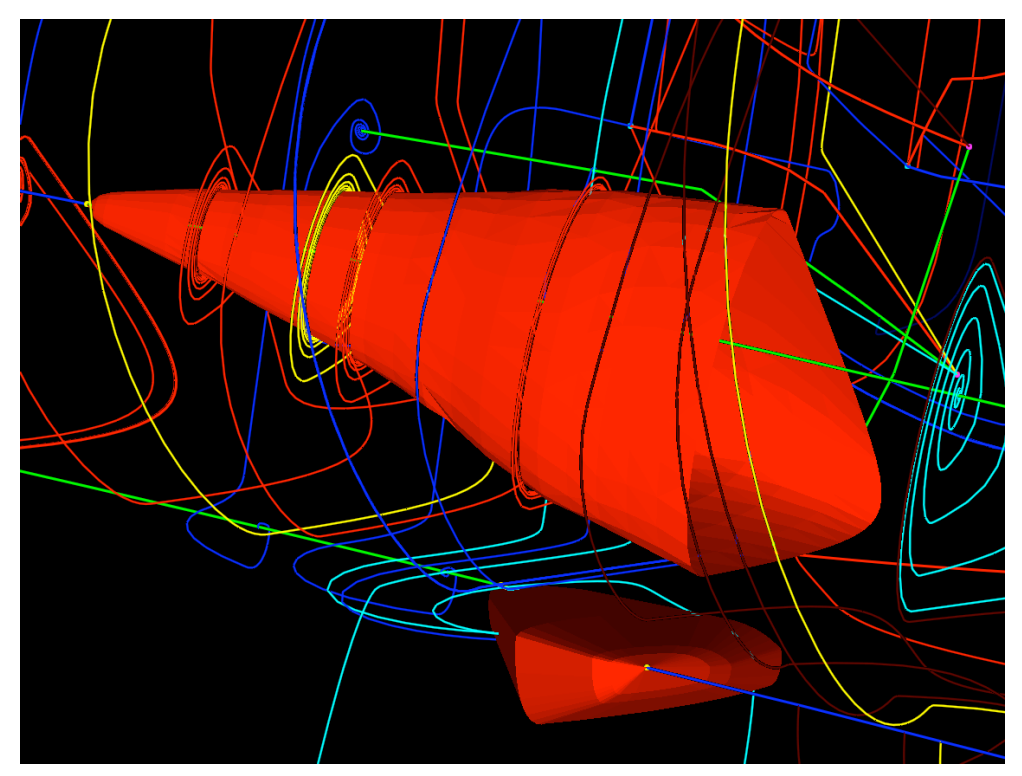

Fig. 11. Periodic Blue Sky

\section{Conclusion}

We have presented a topology-based method for the visualization of time-dependent planar vector fields. All constitutive features of the topological graph, i.e. critical points, closed orbits and separatrices, are continuously tracked over time. The time interpolation of the discrete planar data enables the precise detection of local bifurcations that result in dramatic structural changes. Thus, singularity paths and cycle tubes are displayed as well as the bifurcations that affect them. These objects are then connected in a three-dimensional grid by surfaces spanned by the separatrices over time. This provides the complete structure of the visualized topology. This technique offers a general framework for the visualization of parameter-dependent planar vector fields.

\section{References}

[1] Perry A.E., Chong M.S., A Description of Eddying Motions and Flow Patterns using Critical-Point Concepts Annual Review of Fluid Mechanics 1987, 19, pp. 125-155, 1987.

[2] Abraham R.H., Shaw C.D., Dynamics The Geometry of Behavior, Part I-IV. Aerial Press, Santa Cruz, CA, 1982-1988.

[3] Helman J.L., Hesselink L., Representation and Display of Vector Field Topology in Fluid Flow Data Sets. Visualization in Scientific Computing, G.M. Nielson \& B. Shriver, eds., 1989. 


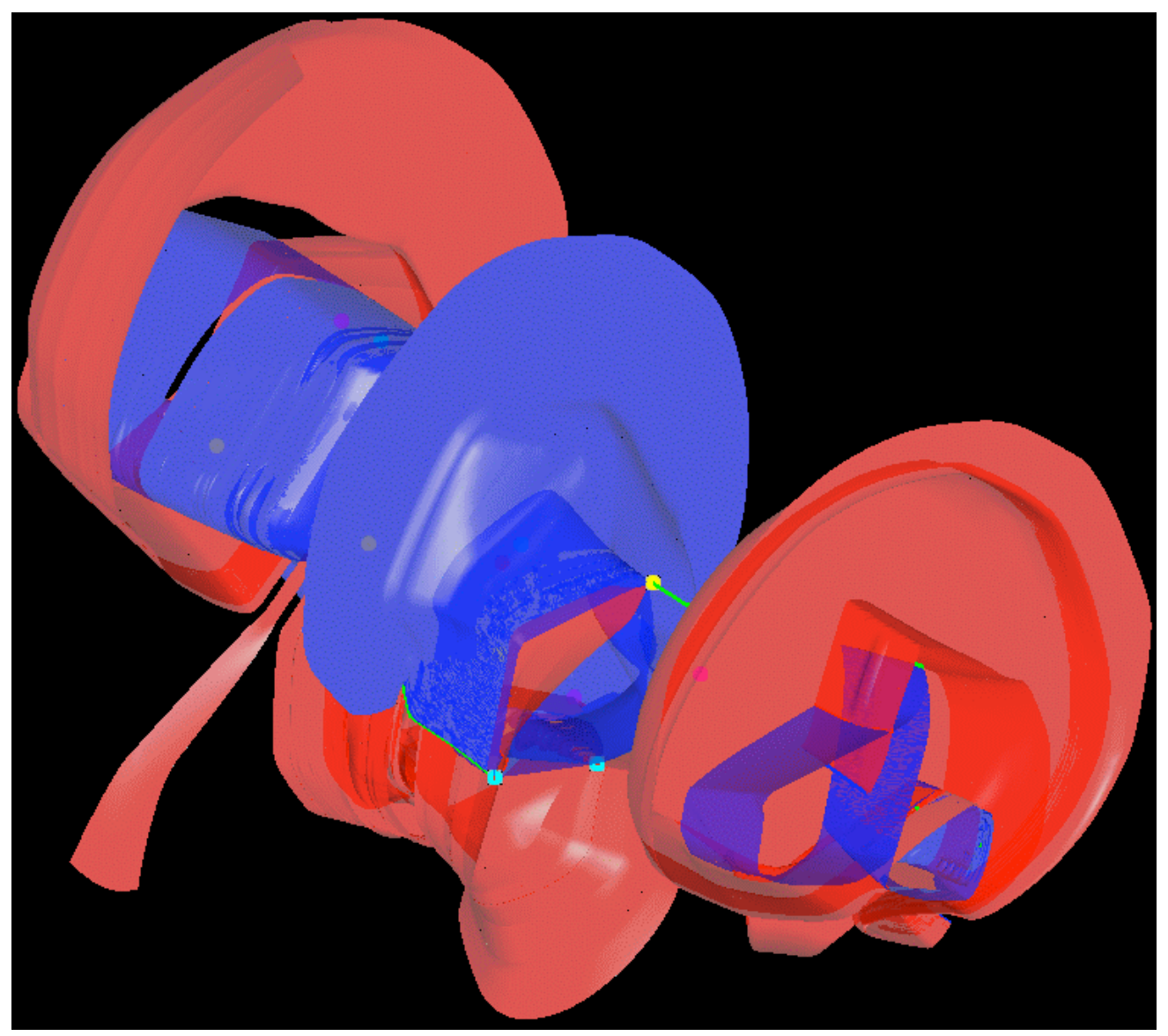

Fig. 12. Overview of the topology evolution

[4] Helman J.L., Hesselink L., Surface Representation of Two- and ThreeDimensional Fluid Flow Topology. Proceedings of the First IEEE Conference on Visualization, pp.6-13, IEEE Computer Society Press, Los Alamitos CA, 1990.

[5] Dickinson R.R., Interactive Analysis of the Topology of 4D Vector Fields. IBM Journal of Research and Development, Vol. 35, No. 1/2, January/March 1991.

[6] Lane D.A., UFAT - A Particle Tracer for Time-Dependent Flow Fields. Proceedings IEEE Visualization'94, IEEE Computer Society Press, Los Alamitos CA, 1994.

[7] Becker B.G., Lane D.A., Max N.L., Unsteady Flow Volumes. Proceedings IEEE Visualization'95, IEEE Computer Society Press, Los Alamitos CA, 1995.

[8] Hepting D.H., Derks G., Edoh D., D. R.R. Qualitative analysis of invariant tori in a dynamical system. In G. M. Nielson and D. Silver, editors, IEEE Visualization '95, pp. 342 - 345, Atlanta, GA, 1995.

[9] Silver D., Feature Visualization In Scientific Visualization Overviews Methodologies - Techniques, pp.279-293, G.M. Nielson, H. Hagen, H. Müller (eds.), IEEE Computer Society, Los Alamitos CA, 1997. 
[10] Wegenkittel R., Löffelmann H., Gröller E. Visualizing the Behavior of Higher Dimensional Dynamical Systems. In R. Yagel and H. Hagen, editors, IEEE Visualization '97, pp. 119 - 125, Phoenix, AZ, 1997.

[11] Scheuermann G., Hagen H., A Data Dependent Triangulation for Vector Fields. In proceedings of Computer Graphics International 1998, pp.96-102, F.E. Wolter, N. M. Patrikalakis (eds.), IEEE Computer Society Press, Los Alamitos CA, 1998.

[12] Wischgoll T., Scheuermann G., Detection and Visualization of Closed Streamlines in Planar Flows. IEEE Transactions on Visualization and Computer Graphics, 7(2), 2001.

[13] Tricoche X., Scheuermann G., Hagen H., Topology-Based Visualization of Time-Dependent $2 D$ Vector Fields. In D. Ebert, J.M. Favre, R. Peikert (eds.), Proceedings of the Joint Eurographics - IEEE TVCG Symposium on Visualization, pp. 117-126, Springer-Verlag, 2001. 